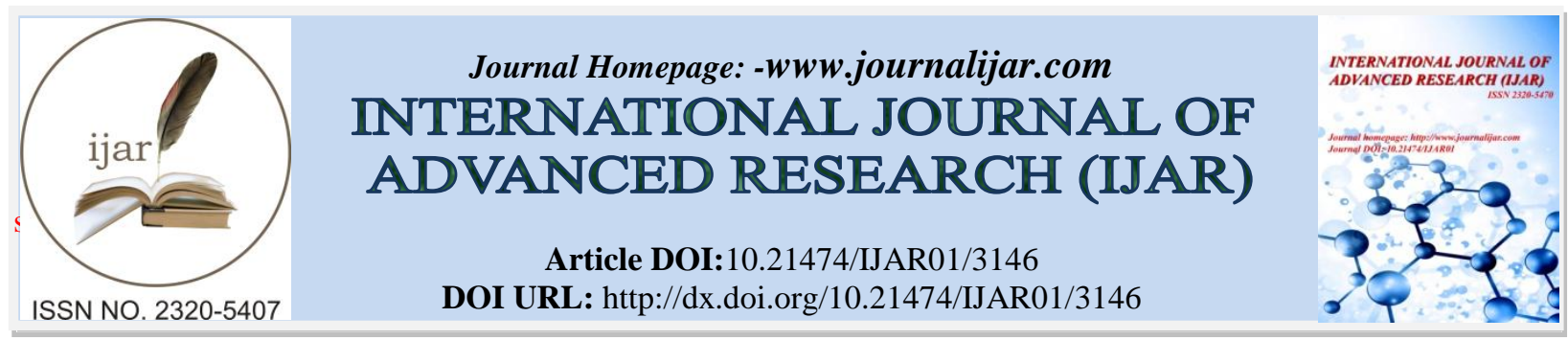

RESEARCH ARTICLE

\title{
A STUDY OF DIFFERENT PHENOTYPIC PRESENTATIONS OF POLYCYSTIC OVARIAN SYNDROME: AN INSTITUTIONAL EXPERIENCE
}

Dr. Malavi ka J.C MS, DNB (OBG).

Assistant professor, Department of Obstetrics and Gynecology, S.S.Institute of Medical Sciences and Research Centre, Davangere, Karnataka, India.

\section{Manuscript Info}

Manuscript History

Received: 13 December 2016

Final Accepted: 18 January 2017

Published: February 2017

Key words:-

Polycysticovarian syndrome, phenotype,oligo/amenorrhea,

hyperandrogenism, cardiometabolic risk

\section{Abstract}

Background:Polycystic ovarian syndrome(PCOS) constitutes one of the most common endocrinopathy amongst the reproductive age women.Over the last few decades it has gained a lot of public attention as reflected in various internet sites and newspapers. However, it is a very complex endocrinopathy presenting as a challenge to clinician to make diagnosis and manage appropriately.

Aims: To study the different clinical presentations among women with PCOS and to categorise them into different phenotypes.

Method: An explorative hospital based study was conducted including 120 women with PCOS, during a two year period between Dec.2014 to Dec.2016. Based on history, clinical examination, hormonal evaluation and ultrasound features, all women were categorized into 4 different phenotypes.

Results: Out of 120 women, 29(24.16\%)presented with full blown syndrome(severe PCOS), 34(28.33\%) had oligo/anovulatory PCOS, $25(20.83 \%)$ of them were found to be ovulatory variety and $32(31.17 \%)$ belonged to mild PCOS category.

Conclusion:PCOS is represents a continuum of symptoms. No single test or clinical feature is sine qua non of the syndrome. This study demonstrates the various phenotypic expressions of PCOS and it can be viewed as a lifelong disorder with progession from mild to severe variety later on in life. Approach to each patient should be tailor made.

Copy Right, IJAR, 2017,. All rights reserved.

\section{Introduction:-}

PCOS is an enigmatic disorder with heterogenous features and is one of the most common endocrinopathy in the reproductive age group. Heterogenity of the disease was evident from the very first description by Stein and Leventhal. Among the 7 women described in the original report, a variety of clinical symptoms were observed such as obesity, amennorhoea, acne,hirsuitism and all of them were associated with bilateral enlarged polycystic ovaries. These varying degree of presentation in each case emphasise the phenotypic variability of PCOS and, explains the very fact why it is called syndrome and why not a disease ${ }^{1}$.

A cluster of symptoms define a syndrome, a common etiologic factor cannot be used to explain the varied clinical presentations. In almost every tissue the hormone acts, but at a different rate, which depends on the receptor function 
and post receptor signaling pathways, which accounts to variety of clinical presentations observed in hormonal disorders. This fact becomes further complicated in PCOS wherein more than one hormone is modified. Women with PCOS manifest with hypothalamic-pitutary-ovarian axis abberations, hyperinsulinemia, hyperandrogenism, as well as adipose tissue dysfunctional adipokine secretion, all of which interact in different tissues (muscle, fat,liver and ovaries), thus leading to a variety of phenotypic presentations ${ }^{1,3}$.

The Rotterdham criteria used to diagnose PCOS requires the presence of atleast 2 of the features: oligo/anovulation, clinical / biochemical hyperandrogenism and ultrasound appearance of polycystic ovarian morphology. In 2006, the Androgen Excess Society (AES) pointed out that PCOS is basically anhyperandrogenic disorder and hence demonstration of acne, hirsuitism and /or biochemical hyper-androgenism becomes a sine qua non of PCOS diagnosis. The second criteria required for the diagnosis of PCOS according to AES is either oligo/anovulation or polycystic ovarian morphology 2,5 .

The introduction of different phenotypes based on Rotterdham criteria makes PCOS represent a continuum of symptoms. PCOS may start gradually as a hyperandrogenic disorder and then lead on to anovulation or vice versa.

Aim of the study:-

To study the different clinical presentations among women with PCOS and to categorise them into different phenotypes.

\section{Objectives of the Study:-}

1. To study the most common clinical features among women with PCOS.

2. To categorise them into different phenotypes.

3. To evaluate all women with PCOS for hormonal imbalances.

4. To study the ultrasound morphology in all women suspected of PCOS.

\section{Methods:-}

Type of study: A hospital based explorative study.

Place of study: All women attending gynecology out-patient department at SS Institute of Medical Sciences and Research Centre with the complaints of menstrual irregularities, infertility and features of hyperandrogenism.

Duration of study: For 2 years between December 2014 to December 2016

Sample size: A total of 120 patients were recruited during the study period after taking informed consent.

Inclusion criteria: Those women who were diagnosed with PCOS according to the Rotterdham criteria were recruited irrespective of the age.

Exclusion criteria: Those women with non- ovarian causes of hyperandrogenism and infertility were excluded.

Study procedure: In OPD, a detailed history was taken. History was noted with respect cyclicityof menses, duration of menses and history of amenorrhea was noted. History suggestive of hypothyroidism (excessive weight gain, menstrual irregularities,menorrhagia) hyperprolactinemia (scanty menses, amenorrhea, premenstrual spotting, secretions from breast) were elicited.

General physical examination was done to note the height, weight and BMI. Clinical signs of hyperandrogenism like acne, hirsuitism were noted. Signs of hyperinsulinemiasuch as acanthosisnigricanswas looked for.

Ultrasound evaluation was done in relevant patients and polycystic ovarian morphology was diagnosed when 12 or more follicles were visualized measuring 2 to $9 \mathrm{~mm}$ or when the ovarian volume bigger than $10 \mathrm{ccm}^{4}$.

Routine blood investigations such as complete hemogram, random blood sugar and urine routine examination were done. Hormonal evaluation comprising of serum thyroid stimulating hormone, s.prolactin, serum FSH, serum LH, serum Testosterone was done. 
For women who wished to conceive, serial follicular monitoring was done to document the presence of absence of ovulation. Those women with anovulation were subjected for ovulation induction according to the institution protocols.

\section{Results:-}

Out of 120 women who were recruited for the study, 18 were adolescents and 102 women were more than 20 years of age .Adolescents were studied separately owing to diagnostic difficulties.

Among the adolescent girls, the most common presenting symptom was menstrual irregularity. Out of 18 girls, $9(50 \%)$ of them presented with amenorrhea, $6(33.33 \%)$ presented with oligomenorrhea, $3(16.6 \%)$ presented with regular cycles. All of them were unmarried.On estimation of BMI among adolescent girls, 8(44.4\%) were overweight,4(22.2\%) were obese and $6(33.3 \%)$ were of normal BMI. All of them were subjected to transabdominalsonography and $13(72 \%)$ had polycystic ovarian morphology and for documenting ovulation to categorize into different phenotypes, follicular monitoring was done and only $3(16.6 \%)$ demonstrated ovulation.Hormonal estimation revealed mildly raised serum testosterone ( $>80$ but $<200 \mathrm{ng} / \mathrm{ml}$ )in $8(44.4 \%)$ girls. Serum TSH was raised in $3(16.6 \%)$ girls and serum prolactin was raised in $1(5.5 \%)$ girl who were subsequently treated with appropriate medications. Serum LH was raised in $10(55.5 \%)$ girls.

Among the women aged more than 20 years, 75(73.5\%) belonged to the age group 20-30years and 27(26.47\%) belonged to the age group 30-40 years.89(87.2\%) were married and 13(12.74\%) were unmarried. Out of them,the most common complaint among them was oligomenorrhea(70.58\%) and infertility(83.3\%). Out of 102 women aged above 20yrs, $72(70.58 \%)$ complained of oligomenorrhea, 16(15.23\%) complained of amenorrhea, 14(13.72\%) had regular cycles.74(72.54\%) of them presented with signs of hyperandrogenism (acne, hirsuitism). On estimation of BMI ,65(63.72\%) were found to be overweight, 15(14.7\%) were obese and 22(21.56\%) had normal BMI .On performing ultrasound (trans-abdominal was preferred by unmarried women and transvaginal ultrasound was done in married women ) on all 102 women, 82(80.39\%) demonstrated polycystic ovarian morphology and 20(19.6\%) demonstrated normal ovarian morphology.Hormonal estimation revealed raised serum $\mathrm{LH}$ in $78(76.47 \%)$ women, serum testosterone was mildly raised in 62(60.78\%) women.

Serial follicular monitoring was done in all women with PCOS to document the presence or absence of ovulation. Out of 120, ovulation was documented in $22(21.56 \%)$ of them.

After collecting and analyzing the data, all adolescents and women were assessed for 3 features of PCOS namely, presence of oligo/anovulation, clinical /biochemical evidence of hyperandrogenism and ultrasound appearance of PCOM. The classification of phenotypes was done accordingly, as demonstrated in Table 1:

Table 1:- Categorization of PCOS phenotype according to the mode of presentation:

\begin{tabular}{|l|c|c|c|c|}
\hline PCOS phenotype & Oligo/anovulation & $\begin{array}{l}\text { Clinical/biochemical } \\
\text { hyperandrogenism }\end{array}$ & PCOM on USG & $\mathrm{n}=120$ \\
\hline 1.Severe PCOS & + & + & + & $29(24.16 \%)$ \\
\hline $\begin{array}{l}\text { 2.Oligo/anovulatory } \\
\text { PCOS }\end{array}$ & + & + & - & $34(28.33 \%)$ \\
\hline 3. Ovulatory PCOS & - & + & + & $25(20.83 \%)$ \\
\hline 4. Mild PCOS & + & - & + & $32(31.37 \%)$ \\
\hline
\end{tabular}

\section{Discussion:-}

Over the last few decades, various diagnostic criteria have been put up insearch of ideal diagnostic approach. The following table 2 depicts the array of various defintions available in the literature:

Table 2:- Definitions and diagnostic criteria for PCOS: ${ }^{1,2,7}$

\begin{tabular}{|l|l|}
\hline Definition/ year & Diagnostic criteria \\
\hline NIH/ 1990 & $\begin{array}{l}\text { Requires the simulataneous presence of: } \\
\text { 1.Clinical and /or biochemical hyperandrogenism } \\
\text { 2.Ovarian dysfunction }\end{array}$ \\
\hline ROTTERDHAM(ESHRE/ASRM) / 2003 & $\begin{array}{l}\text { Requires the presence of atleast 2 criteria: } \\
\text { 1. Clinical and /or biochemical hyperandrogenism }\end{array}$ \\
\hline
\end{tabular}




\begin{tabular}{|l|l|}
\hline & \multicolumn{1}{|c|}{$\begin{array}{l}\text { 2. Ovulatory dysfunction } \\
\text { AES/2006 Polycystic ovarian morphology }\end{array}$} \\
& $\begin{array}{l}\text { Requires the presence of hyperandrogenism(clinical and } \\
\text { / or biochemical)and either: } \\
\text { 1. Ovulatory dysfunction }\end{array}$ \\
& $\begin{array}{l}\text { 2. Polycystic ovarian morphology } \\
\text { Androgen Excess and PCOS Soceity/2009 }\end{array}$ \\
& $\begin{array}{l}\text { Requires the simultaneous presence of: } \\
\text { 1.Clinical and /or biochemical hyperandrogenism } \\
\text { 2.Ovarian dysfunction( ovulatory dysfunction and /or } \\
\text { polycystic ovarian morphology) }\end{array}$ \\
\hline
\end{tabular}

ESHRE=European Soceity of Human Reproduction and Embryology, ASRM= American Soceity for Reproductive Medicine, AES= Androgen Excess Soceity

It is important to state here that although various diagnostic criteria are available for the diagnosis of PCOS, other disorders ( Non Classical Congenital Adrenal Hyperplasia, Cushing's syndrome, acromegaly,hypothyroidism, hyperprolactinemia, virilising adrenal or ovarian neoplasm, premature ovarian failure ) which have overlapping clinical features needs to be excluded ${ }^{8}$.

The ultrasound definition of polycystic ovarian morphology is the presence of $>/=12$ follicles with a 2-to 9- mm diameter on the ovary. Ovarian volume of $>10 \mathrm{ml}$ is also suggestive. One ovary consistent with polycystic ovarian morphology is sufficient for the diagnosis ${ }^{4}$. Although various studies have shown that polycystic ovarian morphology may also be found in $20-30 \%$ of normally ovulating women.

Four different phenotypes of PCOS have now been identified using the possible combinations of these criteria ${ }^{9,10}$ :

- Type A: hyperandrogenism, chronic anovulation and polycystic ovaries.

- Type B: hyperandrogenism and chronic anovulation.

- Type C: hyperandrogenism and polycystic ovaries.

- Type D: chronic anovulation and polycystic ovaries

No single test is considered gold standard for the diagnosis of polycystic ovarian syndrome. A multimodality approach is required to diagnose the syndrome of polycystic ovaries.

The diagnosis of PCOS is all the more challenging in adolescent girls owing to menstrual irregularity due to anovulation which is commonly observed for few years after the onset of menarche.A high index of suspicion needs to maintained when an adolescent persists to have menstrual irregularity for more than two years after menarche. With respect to features of hyperandrogenism in adolescents, slight acne and hirsuitism is an anticipated problem because of transient functional hyperandrogenism and hence it is not of clinical significance. Hence, the combination of ultrasound and androgen evaluation will help to arrive at the diagnosis in an adolescent ${ }^{6}$.

Analysis of the study done reveals that PCOS can manifest with spectrum of symptoms, with the commonest among all age group being menstrual irregularity presenting as oligomenorrhea. Further, infertility is commonly seen among the reproductive age group women owing to anovulation. Hyperandrogenismis considered as the cardinal feature by AES criteria and it was demonstrated in women in our study. An ultrasound demonstration of polycystic ovarian morphology was seen in 99 (82.5\%) women. An ultrasound appearance of PCOM is the result of chronic anovulation, one of the chief causes being PCOS. Other causes of chronic anovulation also needs to be excluded at this point.

The variability of presentation of women with PCOS, with some of them having full blown syndrome(severe PCOS) to others having only 2 of the 3 features point towards the fact that PCOS is continuum of symptoms. Women with mild or ovulatory PCOS can later on in life progress to develop severe PCOS ${ }^{11}$. A tailor made approach is required towards the diagnosis and management of different phenotypes. Adolescents who present with menstrual irregularity can be managed with oral contraceptives and those who come with acne can be managed with oral contraceptives with or without antiandrogens ${ }^{12}$. Women presenting with infertility as chief complaint benefit from weight reduction and metformin which are known to improve the insulin sensitivity and ovulation induction can be done with 
clomiphene citrate or recombinant FSH. Laparoscopic ovarian drilling may be resorted to in women not responding to ovulation induction drugs.

PCOS is known to be associated with significant cardiometabolic risk like dyslipidemia,hyperglycemia, later on in life. Hence, a clinician should not only address the problem the woman seeks but also advise a woman with PCOS to embrace a healthy lifestyle, healthy eating habits and regular exercise to counter the long term effects of this metabolic syndrome ${ }^{13,14}$.

\section{Conclusion:-}

PCOS is a heterogenouscollection of signs and symptoms, which form a spectrum of disorder, with mild variety seen in some women and full blown disorder seen in others. PCOS is viewed as a lifelong disorder with the syndrome progressing from mild to severe variety later on in life.

No single test forms the gold standard diagnostic test in the diagnosis of PCOS. PCOS is a syndrome and it is a diagnosis of exclusion. Hence, other endocrinopathies such as Non Classical Congenital Adrenal Hyperplasia, Cushing's syndrome, acromegaly, hyperprolactinemia,hypothyroidism,premature ovarian failure, virilizing adrenal or ovarian tumor needs to be excluded.

A tailor made approach needs to under-taken in the management of women presenting with PCOS. Since PCOS is associated with significant cardiometabolic risk in later life, all these women need to be advised to embrace healthy lifestyle, healthy nutritional habits and regular exercise.

\section{References:-}

1. Azziz R CE, Dewailly D, Diamanti-KandarakisE,Escobar-Morreale HF, Futterweit W, Janssen OE, Legro RS, Norman RJ, Taylor AE, Witchel SF: Task Force on the Phenotype of the Polycystic Ovary Syndrome of the Androgen Excess and PCOS Society. The Androgen Excess and PCOS Society criteria for the polycystic ovary syndrome: the complete task force report. FertilSteril 2009;91:456.

2. Rotterdam ESHRE/ASRM-Sponsored PCOS Consensus Workshop Group. Revised 2003 consensus on diagnostic criteria and long-term health risks related to polycystic ovary syndrome (PCOS). Hum Reprod 2004;19:41.

3. Cussons AJ, Bronwyn GA, Stuckey G, Walsh JP, Burke V,Norman RJ: Polycystic ovarian syndrome: marked differences between endocrinologists and gynecologists in diagnosis and management. ClinEndocrinol 2005;62:289.

4. Balen AH, Laven JS, Tan SL, Dewailly D: Ultrasound assessment of the polycystic ovary: international consensus definitions. Hum Reprod Update 2003;9:505-514.

5. Broekmans FJ, Knauff EA, Valkenburg O, Laven JS, Eijkemans MJ, Fauser BC: PCOS according to the Rotterdam consensus criteria: change in prevalence among WHO-II anovulation and association with metabolic factors. Br J ObstetGynecol 2006;113:1210.

6. Ibanez L, de Zegher F, Potau N: Anovulation after precocious pubarche: early markers and time course in adolescence. J ClinEndocrinolMetab 1999;84:2691.

7. Shaw LJ, BaireyMerz CN, Azziz R, Stanczyk FZ, Sopko G, Braunstein GD, Kelsey SF, Kip KE, CooperDehoff RM, Johnson BD, et al: Postmenopausal women with a history of irregular menses and elevated androgen measurements at high risk for worsening cardiovascular event-free survival: results from the National Institutes of Health -National Heart, Lung, and Blood Institute sponsored Women's Ischemia Syndrome Evaluation. J ClinEndocrinolMetab 2008;93:1276.

8. Vermeulen A, Verdonck L, Kaufman JM: A critical evaluation of simple methods for the estimation of free testosterone in serum. J ClinEndocrinolMetab 1999;84:3666

9. Diamanti-KandarakisE, Panidis D: Unravelling thephenotypic map of polycystic ovary syndrome(PCOS): a prospective study of 634 women withPCOS. ClinEndocrinol (Oxf) 2007;67:735.

10. Moran L, Teede H: Metabolic features of the reproductivephenotypes of polycystic ovary syndrome.HumReprod Update 2009;15:477.

11. Diamanti-Kandarakis E, Christakou C, Palioura E, Kandaraki E, Livadas S: Does polycystic ovary syndrome start in childhood? PediatrEndocrinol Rev 2008;5:904.

12. Yildiz BO, Bolour S, Woods A, Moore A, Azziz R: Visually scoring hirsutism. Hum Repr Update 2010; 16:51. 
13. Dilbaz B, Ozkaya E, Cinar M, Cakir E, Dilbaz S: Cardiovascular disease risk characteristics of the main polycystic ovary syndrome phenotypes.Endocrine 2011;39:272.

14. Amato MC, Verghi M, Galluzzo A, Giordano C:Theoligomenorrhoic phenotypes of polycystic ovary syndrome are characterized by a high visceral adiposity index: a likely condition of cardiometabolic risk. Hum Reprod 2011;26:1486. 